\title{
Dynamics of thin filopodia during sea urchin gastrulation
}

\author{
Jeffrey Miller ${ }^{1}$, Scott E. Fraser ${ }^{2}$ and David McClay, ${ }^{1, *}$ \\ ${ }^{1}$ Developmental, Cell and Molecular Biology, Duke University, SRC, Box 91000, Durham, NC 27708, USA \\ 2Division of Biology, Beckman Institute (139-74), California Institute of Technology, Pasadena CA 91125, USA \\ *Author for correspondence: e-mail dmcclay@acpub.duke.edu
}

\section{SUMMARY}

At gastrulation in the sea urchin embryo, a dramatic rearrangement of cells establishes the three germ layers of the organism. Experiments have revealed a number of cell interactions at this stage that transfer patterning information from cell to cell. Of particular significance, primary mesenchyme cells, which are responsible for production of the embryonic skeleton, have been shown to obtain extensive positional information from the embryonic ectoderm. In the present study, high resolution Nomarski imaging reveals the presence of very thin filopodia (0.2-0.4 $\mu \mathrm{m}$ in diameter) extending from primary mesenchyme cells as well as from ectodermal and secondary mesenchyme cells. These thin filopodia sometimes extend to more than $80 \mu \mathrm{m}$ in length and show average growth and retraction rates of nearly $10 \mu \mathrm{m} /$ minute. The filopodia are highly dynamic, rapidly changing from extension to resorption; frequently, the resorption changes to resumption of assembly. The behavior, location and timing of active thin filopodial movements does not correlate with cell locomotion; instead, there is a strong correlation suggesting their involvement in cell-cell interactions associated with signaling and patterning at gastrulation. Nickel-treatment, which is known to create a patterning defect in skeletogenesis due to alterations in the ectoderm, alters the normal position-dependent differences in the thin filopodia. The effect is present in recombinant embryos in which the ectoderm alone was treated with nickel, and is absent in recombinant embryos in which only the primary mesenchyme cells were treated, suggesting that the filopodial length is substratum dependent rather than being primary mesenchyme cell autonomous. The thin filopodia provide a means by which cells can contact others several cell diameters away, suggesting that some of the signaling previously thought to be mediated by diffusible signals may instead be the result of direct receptor-ligand interactions between cell membranes.

Key words: primary mesenchyme, secondary mesenchyme, cell interactions, videoimaging, nerve growth cone, sea urchin

\section{INTRODUCTION}

During gastrulation of the sea urchin embryo, a variety of cell interactions play critical roles in patterning and the assignment of cell fate. For example, 64 primary mesenchyme cells (PMCs) synthesize a $\mathrm{CaCO}_{3}$ skeleton in a highly organized pattern that is species-specific. A number of experiments involving transplantation, augmentation, depletion or movement of PMCs have shown that the pattern of skeletogenesis requires an interaction between the PMCs and the overlying ectoderm (Ettensohn and McClay, 1986; Ettensohn, 1990b; Hardin et al., 1992; McClay et al., 1992; Armstrong et al., 1993; Armstrong and McClay, 1994). This information is necessary for the PMCs to acquire axial, temporal and scalar information; without which the skeleton produced has no predictable pattern. In other interactions, PMCs prevent secondary mesenchyme cells (SMCs) from changing lineage and assuming a PMC phenotype. If PMCs are removed from embryos, SMCs convert to replace the missing PMCs (Ettensohn and McClay, 1988). These interactions can occur over some distance between cells that are not near neighbors; and, therefore, require signaling systems that can facilitate the transfer of patterning information at a distance.

During gastrulation and preceding skeletogenesis, the PMCs and SMCs undergo a dynamic series of cell movements. Before they begin to make the skeleton, the PMCs move along the inner wall of the blastocoel and organize into a ring surrounding the archenteron. Independently, the SMCs arise from the lead end of the archenteron, eventually leaving to become pigment cells, blastocoelar cells, cells of the coelomic pouches and muscle cells lining the foregut. All of these movements can be observed in vivo with light microscopy because of the relative transparency of the overlying ectoderm. In time-lapse studies performed a number of years ago, Gustafson and colleagues described the movement of PMCs and SMCs including their active extension of filopodia (Gustafson and Wolpert, 1961, 1967; Gustafson, 1963, 1964). SMCs at the tip of the archenteron were observed to extend filopodia throughout gastrulation. Filopodial contacts with ectoderm were made as PMCs migrated along the wall of the blastocoel. Gustafson's analysis centered upon the fairly thick $(1 \mu \mathrm{m}$ in diameter or larger) filopodia, although he briefly alluded to what appeared 
to be thinner processes that he termed 'pseudopodia', which were below the limits of his ability to resolve. Based on their behavior and distribution, Gustafson proposed both sensory and mechanical roles for the filopodia. More recent experimental analyses have confirmed both a mechanical role and a cell-cell interactive function of the thick filopodia. During the last third of archenteron extension, filopodia extending from SMCs assist in pulling the archenteron to its final length (Hardin and Cheng, 1986; Hardin, 1987, 1988). Thick filopodia also play a role in positional signaling since their movements ultimately result in recognition and adhesion to a target site that places the archenteron in its final anatomical position (Hardin and McClay, 1990).

But what are the functions of the thin filopodia? The thin filopodia ('pseudopodia' of Gustafson) have been less thoroughly examined beyond occasional reports of their presence. Ultrastructural studies of sea urchin embryos reported their presence (Gibbins et al., 1969; Tilney and Gibbins, 1969) and in vitro studies documented thin filopodia extending from PMCs (Karp and Solursh, 1985). As a result, Karp and Solursh hypothesized that the thin filopodia might be involved in the movement of PMCs during their exploratory behavior prior to skeletogenesis, or that they may be involved in forming the syncytium that joins PMCs together during skeletogenesis.

Here we draw upon recent advances in videomicroscopy and image processing to investigate the thin filopodia in vivo in the sea urchin embryo. We find that the thin filopodia are much more common than previously believed, and that they show complex and highly dynamic behavior as they are extended by PMCs, SMCs and ectoderm. We confirmed our video observations by using immunofluorescence with antibody probes that recognize the cell surface and filopodial extensions of PMCs. The timing and pattern of these thin cellular protrusions correlates well, not with their use in locomotion of cells, but with their use in the exchange of the patterning information used by the PMCs and SMCs. As a test of this hypothesis, we imaged the filopodia following the treatment of embryos with $\mathrm{NiCl}_{2}$. Earlier transplantation experiments showed that nickel disrupts skeletal patterning by altering the dorsal-ventral axis in the ectoderm without showing any obvious direct effect on PMCs (Hardin et al., 1992; Armstrong et al., 1993). Here, we find that the thin filopodia are abnormally long in nickel-treated embryos and in recombinants in which the ectoderm had been nickel-treated. The abnormal filopodia in these embryos with disrupted positional cues, as well as our earlier observations on the distribution and dynamics of the thin filopodia, suggest that the filopodia play a sensory, not a mechanical, role in embryogenesis.

\section{MATERIALS AND METHODS}

\section{Embryos}

Adult Lytechinus variegatus animals were obtained from Susan Decker Services, North Hollywood, FL. Gametes were obtained by intracoelomic injection of $0.5 \mathrm{M} \mathrm{KCl}$. Eggs were dejellied by passage through several layers of cheesecloth and fertilized with a dilute sperm suspension. Embryos were grown in filtered seawater.

\section{Microscopy}

Embryos were mounted between poly-L-lysine-coated coverslips and slides using $100 \mu \mathrm{m}$ thickness double-stick tape as coverslip supports to prevent crushing of the embryos. Coverslips were sealed with Wesson oil to prevent evaporation of sea water. The thin filopodia were observed with a Zeiss Axioplan microscope, equipped with Nomarski differential interference contrast (DIC) optics, oilimmersion condenser and high N/A oil Plan-Apo or Plan-Neofluar objectives. Depending upon the stage of the embryo and nearby features, contrast from the thin filopodia was maximized by setting the DIC optics either very near extinction or far from extinction, and/or by closing the condenser aperture slightly. Illumination was from a $100 \mathrm{~W}$ quartz halide incandescent bulb, through a green interference filter. Typically, a $63 \times$ objective was used in conjunction with a zoom Optivar, which magnified the image projected onto the Newvicon videocamera (Hamamatsu). The output from videocamera was processed (analog contrast enhancement, image averaging, background subtraction, histogram stretching) using an Imaging Technologies 151 processor controlled by the VidIm software package (Belford, Stollberg \& Fraser, unpublished), which allowed for collection of contrast-enhanced image sequences. Individual images were stored digitally onto removable media (Bernoulli); time-lapse sequences were stored in analog form onto a laser-disc recorder (OMDR; Panasonic). Measurements of filopodial length, and growth and retraction rates were made from stored images in which the entire length of a filopodium could be followed. Measurements are given as the mean \pm standard deviation.

\section{Labeling}

For immunofluorescence, embryos were fixed 20 minutes in methanol at $-20^{\circ} \mathrm{C}$. They were incubated with monoclonal antibodies Ig8 or Id5, both of which recognize a cell surface antigen, msp130, that is specific for PMCs (Leaf et al., 1987; McClay et al., 1983). Following several washes in sea water, the embryos were incubated in a 1:100 dilution of Cy3-conjugated goat anti-mouse IgG (Jackson Labs). Embryos also were labeled in some experiments with DiI (Molecular Probes) by touching cells with a needle coated with deposits of DiI crystals. By holding the coated glass needle against the embryo for several minutes, the lipophilic DiI is transferred to the membrane of targeted cells, allowing them to be viewed with a rhodamine fluorescence filter set. Actin filaments were stained with Rhodamine Phalloidin (Molecular Probes). The PMCs were stained by co-incubating the cells in rhodamine phalloidin $(0.01 \%)$ as the cells were fixed in $3.7 \%$ formalin in artificial sea water for 20 minute.

\section{Inhibitors}

Embryos were incubated in either $\beta$-amino proprio nitrile ( $\beta$-APN, 0.2-0.4 mM; Sigma) or $\mathrm{NiCl}_{2}(0.5-1.0 \mathrm{mM})$ for the times and concentrations indicated, after which the inhibitors were washed away. At the concentrations used, both inhibitors affect only specific events at specific stages during development. $\beta$-APN reversibly blocks PMC migration and archenteron invagination (Wessel and McClay, 1987), and $\mathrm{NiCl}_{2}$ abolishes the dorsal-ventral axis (Hardin et al., 1992).

\section{Cell transfers}

Embryos were grown at room temperature either in artificial seawater (ASW) plus $\mathrm{NiCl}_{2}(1 \mathrm{mM})$ for $8-10$ hours and then washed into ASW alone. Prior to doing cell transfers nickel-treated embryos or control embryos were incubated for 1 hour in ASW containing RITC to stain the donor cells (Ettensohn and McClay, 1986). PMCs were transferred from RITC-labeled donors to unlabeled host embryos as previously described (Ettensohn and McClay, 1986).

\section{RESULTS}

Through a combination of Nomarski differential interference contrast microscopy and electronic image enhancement, thin filopodia can be observed extending from several cell types 
beginning at early gastrulation. The sea urchin gastrula (Fig. 1) is an ideal preparation for observing these filopodia in vivo because of the thin layer of transparent ectoderm surrounding the largely featureless blastocoel. The thin filopodia extend from the basal end of ectodermal cells (Fig. 2), from primary mesenchyme cells (PMCs; Figs 2, 3) and from secondary mesenchyme cells (SMCs; see below), throughout gastrulation. A thin basal lamina lines the blastocoel separating the ectoderm on the outside from the PMCs and SMCs on the inside. The basal lamina serves as the major substratum for PMC migration, but does not appear to impede the filopodia. Ectodermal cells on one side of the basal lamina extend filopodia through and into the blastocoel. PMCs, in turn, extend thin filopodia that often make direct contact with the ectoderm.

The thin filopodia extended by ectoderm are short $(5-10 \mu \mathrm{m})$ relative to those extended by PMCs (Fig. 2; see quantification below). Ectodermal filopodia also seem to be short lived relative to the lifetime of the filopodia extended by PMCs and SMCs. Typically, an ectoderm cell extends only a single thin filopodium into the blastocoel at any given time. Because of their short life and the small minority of the ectoderm cells that extend filopodia at any one time, these filopodia were not studied in any further detail.

PMCs extend thin filopodia throughout gastrulation but the greatest abundance of thin filopodia appears only after the period of active PMC migration (Figs 2, 3). At the beginning of gastrulation, PMCs ingress into the blastocoel and then migrate along the walls of the blastocoel for several hours before gathering into a ring around the lower circumference of the growing archenteron (Fig. 3). During their migratory phase PMCs extend few, if any, thin filopodia (Fig. 3A,B). After these cells stop moving, they gather into the subequatorial ring to organize the prepattern of the larval skeleton. Two ventrolateral clusters of PMCs initiate the synthesis of the triradiate spicule rudiments that will continue to grow within a syncytial cable between PMCs. Thin filopodia become abundant as the

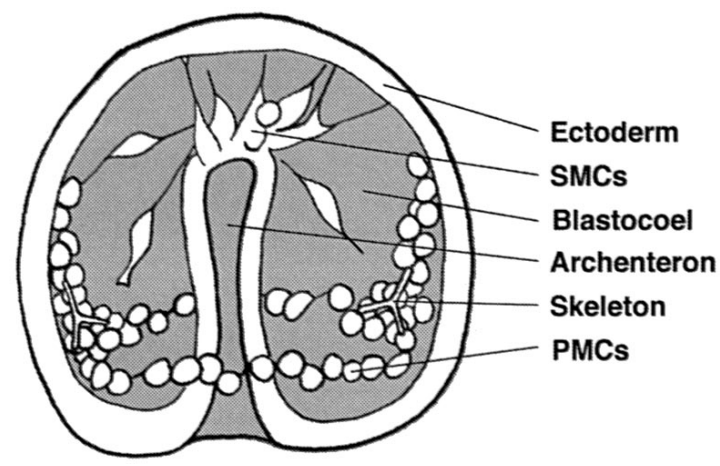

Fig. 1. Diagram of a midgastrula stage sea urchin embryo shown to point out the structures covered in this paper. The ectoderm is a monolayer of cells that surrounds the central cavity called the blastocoel. Lining the blastocoel is a thin basal lamina. The archenteron or primitive gut is in the process of invaginating from the vegetal pole toward the animal pole. At the leading tip of the archenteron secondary mesenchyme cells (SMCs) extend long thick filopodia that make contact with the wall of the blastocoel. Primary mesenchyme cells (PMCs) at this stage surround the base of the archenteron in a ring. At two points along the ring, they initiate the synthesis of the larval skeleton. Not shown are the many thin filopodia that extend from PMCs, SMCs and ectoderm at this time.
PMCs coalesce into the ring and continue to be extended throughout the period of spiculogenesis (Fig. 3C-I). PMCs usually extend thin filopodia along the wall of the blastocoel in all directions. Some filopodia extend up into the blastocoel, presumably along the virtually invisible collagen cables that cross the blastocoel cavity. Thin filopodia make frequent contact with the ectodermal cells and with SMCs that are also within the blastocoelar cavity. Most of the thin filopodia grow and retract without an observable change in their diameter (see below), although occasionally one can observe a filopodium develop a lamellar thickening (Fig. 3D,E). Often, single PMCs extend 5-10 filopodia concurrently (Fig. 3F-H). Such multiple filopodia sometimes extend from a short lamellipod-like protuberance, not from the body of the cell itself. Thin filopodia are especially active and abundant on cells at the lead end of the longitudinal cell chain where they sometimes reach lengths of $>80 \mu \mathrm{m}$ (Fig. 2). Although PMCs are relatively immobile during spiculogenesis, filopodial behavior is very dynamic at this time (Fig. 4; see below). The lifetime of individual filopodia averaged 6 minutes, although this was highly variable, with some filopodia lasting greater than 30 minutes and others retracting after only about 2-3 minutes of extension.

The SMC population of cells are highly variable in function and behavior. SMCs first appear at the tip of the invaginating archenteron where they extend long thick filopodia across the blastocoel to make contact with the basal lamina some 30-35 $\mu \mathrm{m}$ away. This behavior is exhibited during the entire period of archenteron elongation until the tip of the archenteron finally makes contact with a target area near the animal pole, an area that will eventually fuse with the ectoderm to form the stomodaem. The diverse group of cells supplied to the embryo

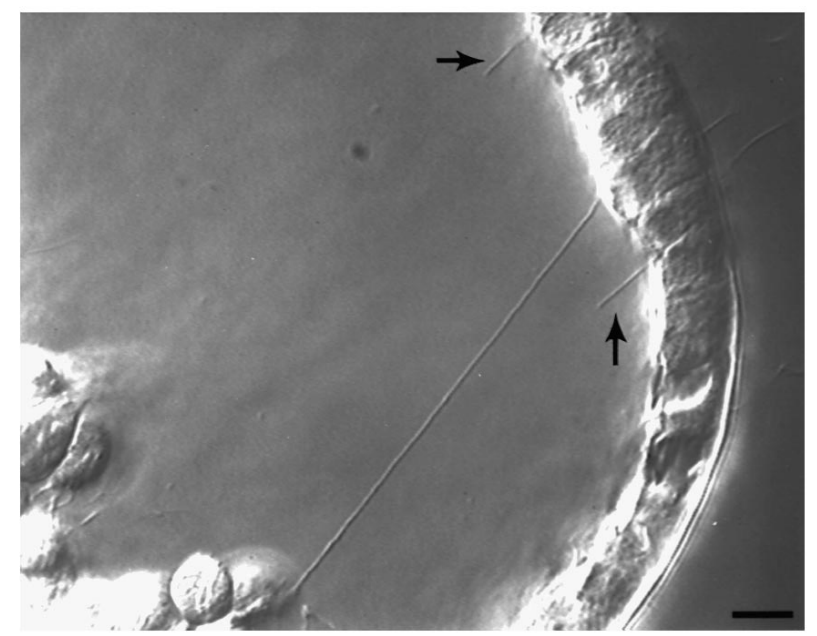

Fig. 2. Videomicrograph demonstrating the presence of ectodermal filopodia and extremely long primary mesenchyme cell filopodia. A long thin filopodium is seen extending from a PMC to the wall of the ectoderm about $80 \mu \mathrm{m}$ away. The PMC is located at the tip of the longitudinal cell chain and the filopodium extends almost to the animal pole. This filopodium measures about $0.2-0.4 \mu \mathrm{m}$ in diameter and its diameter changes little over its entire length. Also seen are thin filopodia extending from ectoderm cells into the blastocoel (arrows). The ectoderm cells are partially obscured by a thin basal lamina that lines the blastocoel. The basal lamina does not prevent ectodermal filopodia from extending into the blastocoel and its thin mesh-like structure does not prevent PMC filopodia from making direct contact with ectodermal cell membranes. Scale bar, $10 \mu \mathrm{m}$. 

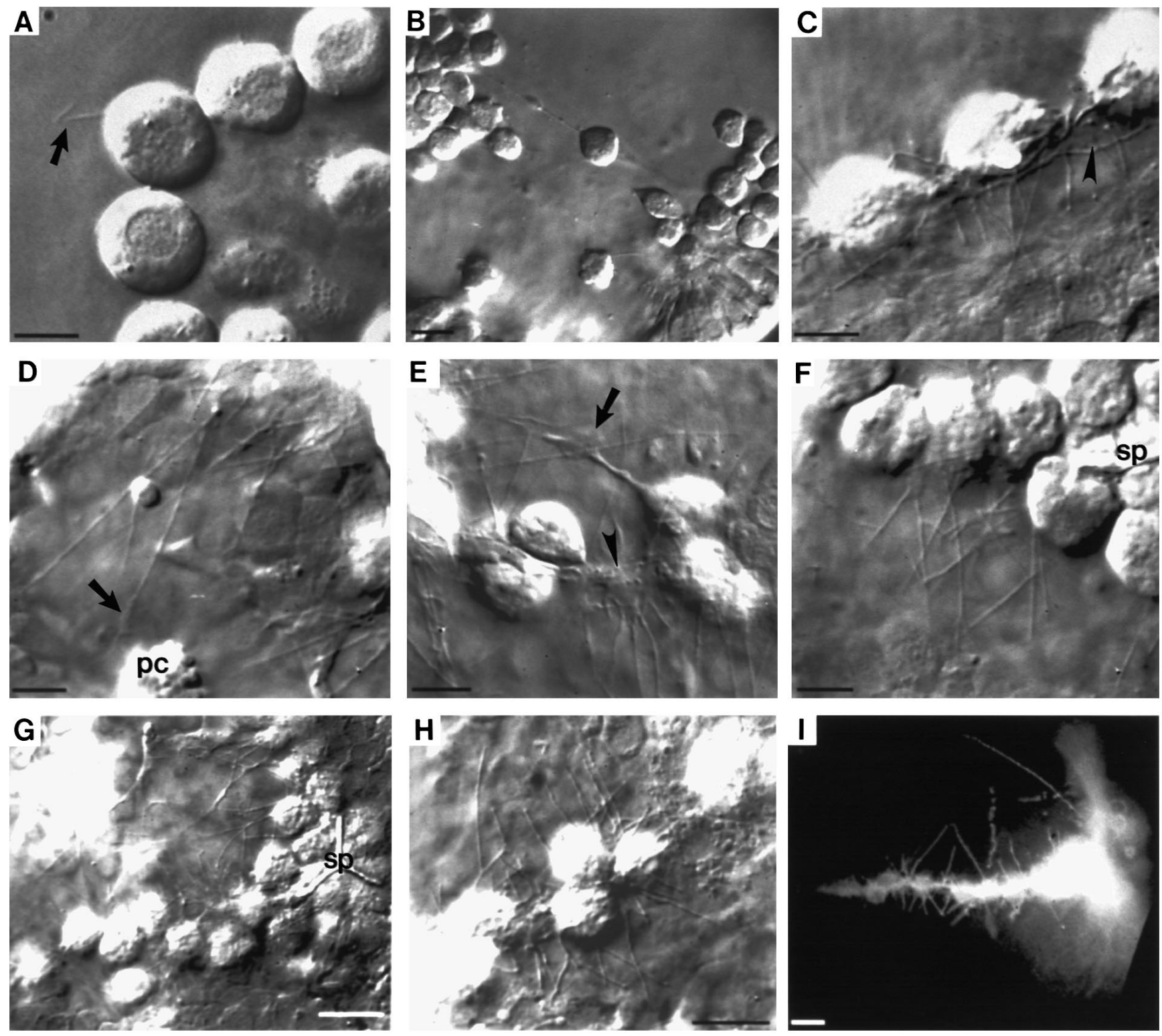

Fig. 3. Videomicrographs illustrating the characteristics of thin filopodia extended by primary mesenchyme cells during gastrulation. Early in gastrulation, just after the PMCs ingress they are highly motile but extend few thin filopodia (arrow in A). Many PMCs initially migrate toward the animal pole, but then turn around and go back toward the vegetal plate where they form a ring surrounding the invaginating archenteron (B). All during this time, there are few filopodia extended. The ring of PMCs then join together to form a syncytium as seen by the cable-like membrane fusions (arrowhead in $\mathrm{C}$ and $\mathrm{E}$ ). At this time, the first skeletal elements begin to be synthesized and the period of enhanced filopodial extension begins. Although most thin filopodia are simple rods, occasionally a portion of the filopodium flattens at its tip (D,E). Filopodial activity is abundant in the region of spicule growth $(\mathrm{F}, \mathrm{G})$ and filopodial lengths differ at different points in the ring and on the growing spicule. Each PMC extends multiple thin filopodia in all directions along the basal surface of the ectoderm (H). In I, a group of PMCs were labeled with diI, a lipophilic dye that occupies membranes. The thin filopodia are seen projecting from a portion of the PMC ring. sp, spicule; pc, pigment cell. Scale bars: $5 \mu \mathrm{m}(\mathrm{A}, \mathrm{C}-\mathrm{F}, \mathrm{H}), 10 \mu \mathrm{m}(\mathrm{B}, \mathrm{G}, \mathrm{I})$.

by the pleuripotent SMCs often are highly migratory. At times of rapid migration, SMC family members extend thick filopodia that appear to be utilized in the translational motility of these cells. At the same time, these cells extend thin filopodia that make frequent contact with the basal lamina and an extensive series of interactions with PMCs (Fig. 5). Often the SMC filopodia switch from one phenotype to another; a thin filopodium will thicken, apparently by a flow of material into it from the cell body; the thicker filopodium often becomes a lamellipodium (Fig. 5C). This change in the phenotype of cellular extensions occurs far more frequently on SMCs than it does on PMCs. Thin filopodia extended by SMCs display dynamics similar to the PMCs in terms of growth and retraction rates. They tend, however, to be shorter and fewer in number on each cell.

\section{Characterization of thin filopodia}

A number of measurements were made on numerous filopodia 

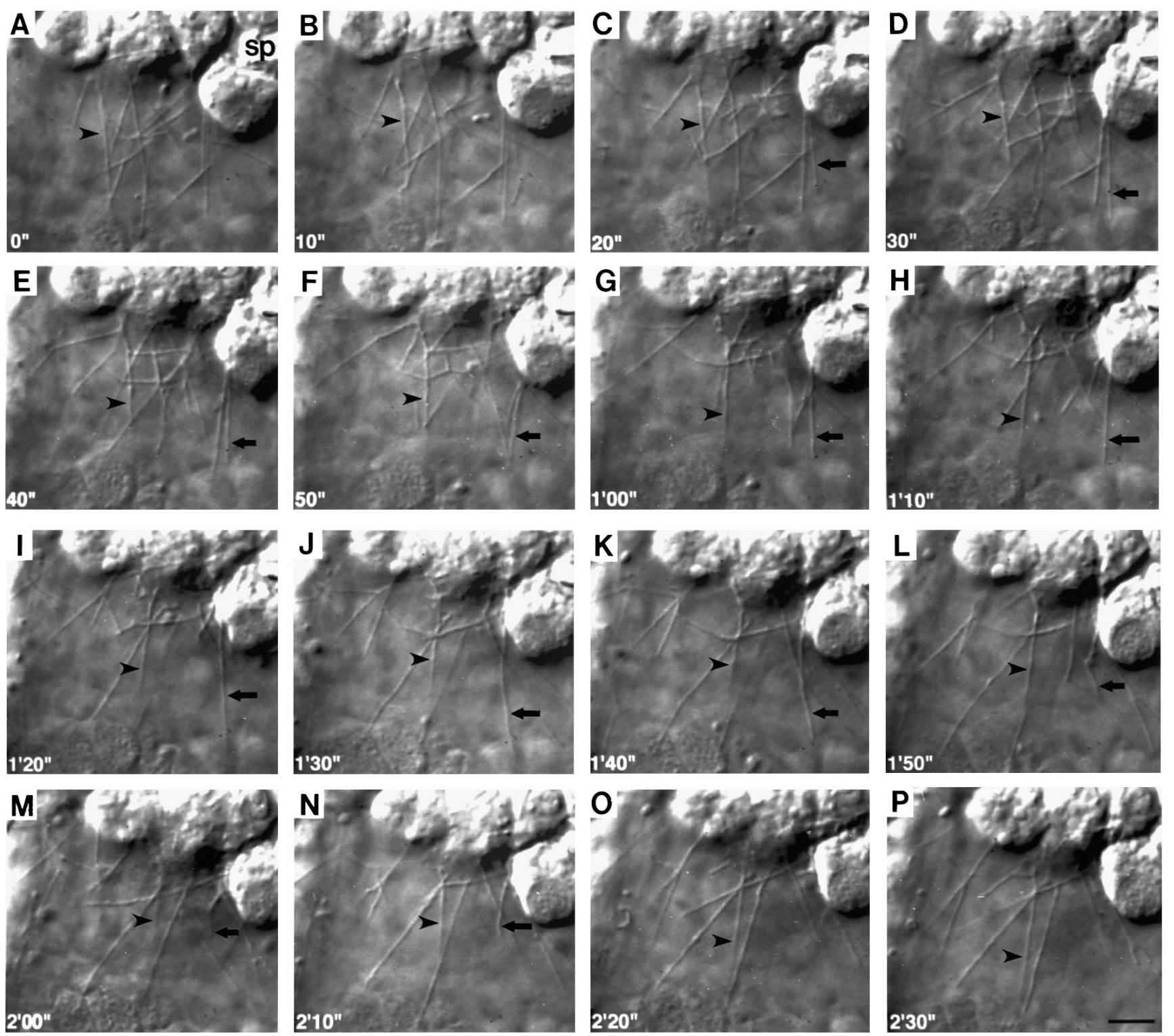

Fig. 4. Time-lapse sequence demonstrating the dynamics of primary mesenchyme cell filopodial activity (A-P). This sequence was taken in a region in the subequatorial ring just ahead of the growing spicule ( $\mathrm{sp}$ in A). An arrowhead follows a filopodium present throughout the 2.5 minute sequence. This filopodium appears to make contact with both ectoderm cells and with other thin filopodia. An arrow follows a filopodium that first appears in B, extends to contact several ectoderm cells (D-K) and is eventually retracted (L-N). Note how this filopodium kinks as it is retracting (L,M). Time elapsed from the first image is shown in the lower left hand corner in minutes and seconds. Scale bar $=5 \mu \mathrm{m}$.

to characterize their dynamics. Extension of thin filopodia occurs rapidly $(8.6 \pm 3.6 \mu \mathrm{m} /$ minute; $n=60$; Fig. 6$)$. The fastest growth observed was $25.2 \mu \mathrm{m} /$ minute. Bouts of rapid growth are sometimes interrupted by periods of inactivity, during which the filopodium simply stops growing for a short time (Fig. 6) after which it resumes growth. As the thin filopodium grows, its diameter changes very little along its entire length. The diameter appears to be about $0.2-0.4 \mu \mathrm{m}$, although the uniformity in the diameter of a thin filopodia is sometimes interrupted by a bulge that travels towards the cell body. Absolute measures of the diameters are difficult because of not only the resolution limits of the light microscope but also the optical and imaging methods that were used to enhance contrast at the expense of some resolution. Nevertheless, these videomicroscopy measurements are in rough agreement with ultrastructural measurements (Gibbins et al., 1969; Tilney and Gibbins, 1969). Resorption of the filopodia also occurs rapidly (11.4 $\pm 5.1 \mu \mathrm{m} /$ minute, $n=27$; Fig. 7). The fastest retraction observed took place at $24 \mu \mathrm{m} /$ minute. Often filopodia are observed to 'kink' at some point along their length, usually during or just preceding resorption, a behavior termed 'jackknifing' in previous studies (Sheetz et al., 1992). Resorption is not an irreversible process; many filopodia, after being partially resorbed, renew a phase of extension.

From video sequences in which the entire length of a filopodium was in the plane of focus, more than 250 filopodia 

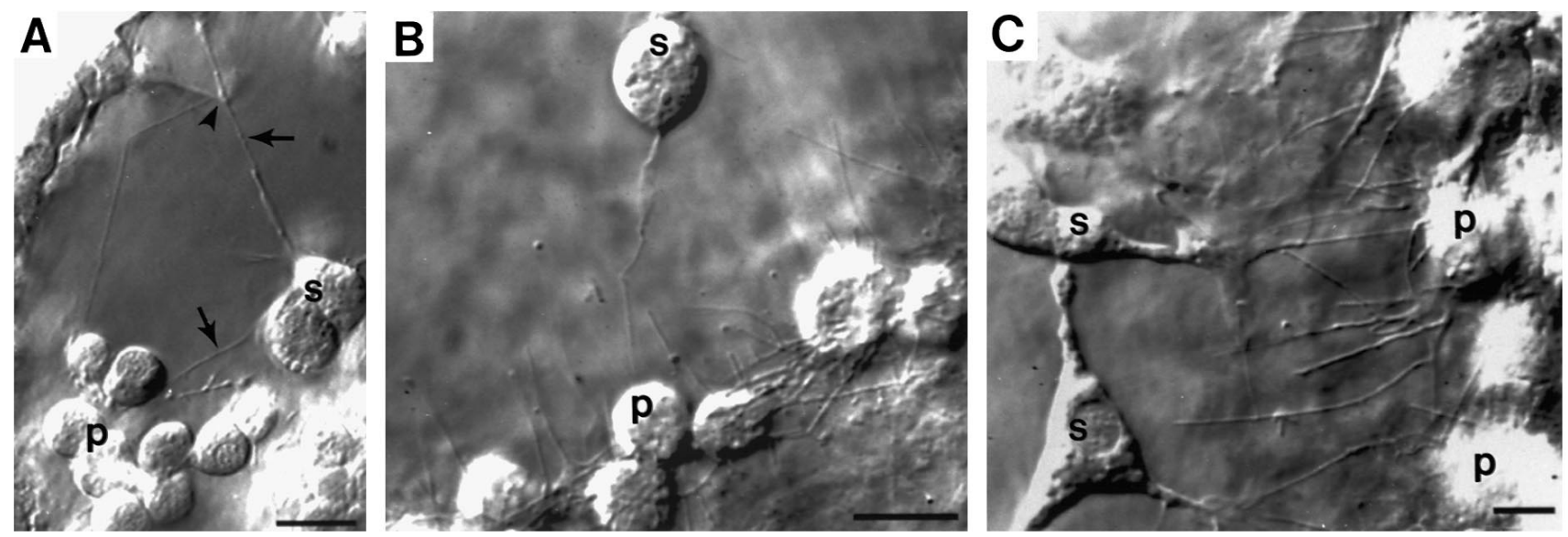

Fig. 5. Videomicrographs showing the characteristics of secondary mesenchyme cell filopodia. In A, SMCs (s) are seen to extend thin filopodia (arrows) that touch PMCs (p) or that extend toward the wall of the blastocoel. PMCs also extend filopodia to touch SMCs (arrowhead). These interactions appear to be necessary to prevent an SMC to PMC lineage conversion which can be induced to occur if the PMCs are removed from the blastocoel. In B, an SMC is touching a PMC. This filopodium is complicated, being thin at the end with a lamellar portion near the cell body. In C, SMCs are extending thick filopodia, thin filopodia and lamellipodia. Also, PMCs are touching the SMCs at many points along their length. Scale bars: A,B, $10 \mu \mathrm{m}, \mathrm{C}, 5 \mu \mathrm{m}$.

were scored for length (Table 1). The average length of the thin filopodia extended by PMCs was found to differ according to the location of the PMCs within the embryo. In the subequatorial ring, PMCs extend filopodia that average $12 \mu \mathrm{m}$ in length. PMCs of the ventral lateral clusters extend thin filopodia that average $17 \mu \mathrm{m}$ in length, while cells at the tips of the longitudinal chains extend filopodia that are on average $>20 \mu \mathrm{m}$ long. Occasionally a filopodium can grow to greater than $80 \mu \mathrm{m}$ in length. When such filopodia were observed, they were found to extend only from PMCs at the tips of the longitudinal cell chain and never from PMCs within the ring or ventral lateral clusters.

\section{Composition of the thin filopodia}

Gustafson and Wolpert (Gustafson, 1963) referred to their 'pseudopodia' as being composed of microtubules. The later in vitro studies of Karp and Solursh (1985), suggested that the thin filopodia might contain actin (based on the collapse of these extensions in cytochalasin). To examine the cytoskeletal content of thin filopodia further, we stained cultured PMCs with rhodamine-conjugated phalloidin, a toxin that binds selectively to actin filaments. As seen in Fig. 8 the thin filopodia are stained, thereby supporting the notion that they contain actin.

\section{Filopodia and cell-cell interactions}

To gain a better insight into the function of thin filopodia, their behavior was followed at different stages of development by time-lapse videomicroscopy. Early migratory PMCs extended very few, if any, filopodia (see Fig. 3A, for example). If the filopodia were being used for cell locomotion, one would expect them to be extended ahead of an advancing cell during the time of cell movement. The filopodium could then attach, exert tension and pull the cell along, perhaps being swept rearward as the cell advanced beyond the original filopodial contact site. When moving cells were observed, however, they extended very few thin filopodia and even fewer of these were in the direction of movement. Cells moving forward were just as likely to extend thin filopodia laterally, or posteriorly, as they were from the leading edge of the cell. Similarly, the movement of PMCs was not observed to be accompanied by a tension-inducing shortening of filopodia. We simply could not find examples of thin filopodia pulling along the cell in a fashion frequently observed when the thick filopodia/lamellipodia of SMCs are followed. Further, cell movement seemed unaffected either by resorption or by extension of the filopodia. Finally, the most extensive filopodial activity began after the cells had stopped moving and gathered into the ring, peaking during the time of skeletogenesis. Thus, it does not seem likely that the thin filopodia are used for locomotion.

If they are not being used for cell motility, for what are the thin filopodia being used? Thin filopodia were prevalent at positions in the embryo and at times when earlier studies showed PMCs interacting either with the ectoderm or with SMCs to transfer patterning or lineage information (Ettensohn and McClay, 1988; Ettensohn, 1990a; McClay et al., 1992; Armstrong et al., 1993; Armstrong and McClay, 1994). The correlation of the thin filopodia with all of these interactions raised the clear possibility that the filopodia were involved in cell-cell signaling.

To test more directly the role(s) of the thin filopodia, they were examined in embryos treated with an inhibitor known to alter dorsoventral pattern formation. Fig. 9 shows confocal images of embryos stained with an antibody that reveals filopodial morphology. The figure shows that the thin filopodia are much longer in $\mathrm{NiCl}_{2}$-treated embryos than in controls (see Table 1 for quantification). Previous studies have shown that nickel treatment eliminates dorsal structures and makes the embryo highly ventralized (Hardin et al., 1992). Reciprocal transfer experiments in which nickel-treated or untreated PMCs were transferred to host embryos containing nickel-treated or untreated ectoderm show that the ectoderm, not the PMCs, is altered by the $\mathrm{NiCl}_{2}$ (Hardin et al., 1992; Armstrong et al., 1993). The finding that thin filopodia are longer after $\mathrm{NiCl}_{2}$ treatment than in controls (sometimes $>100 \mu \mathrm{m}$, Fig. 9) is curious because these previous studies demonstrated that nickel had no direct effect on the ability of PMCs to make a skeleton. 

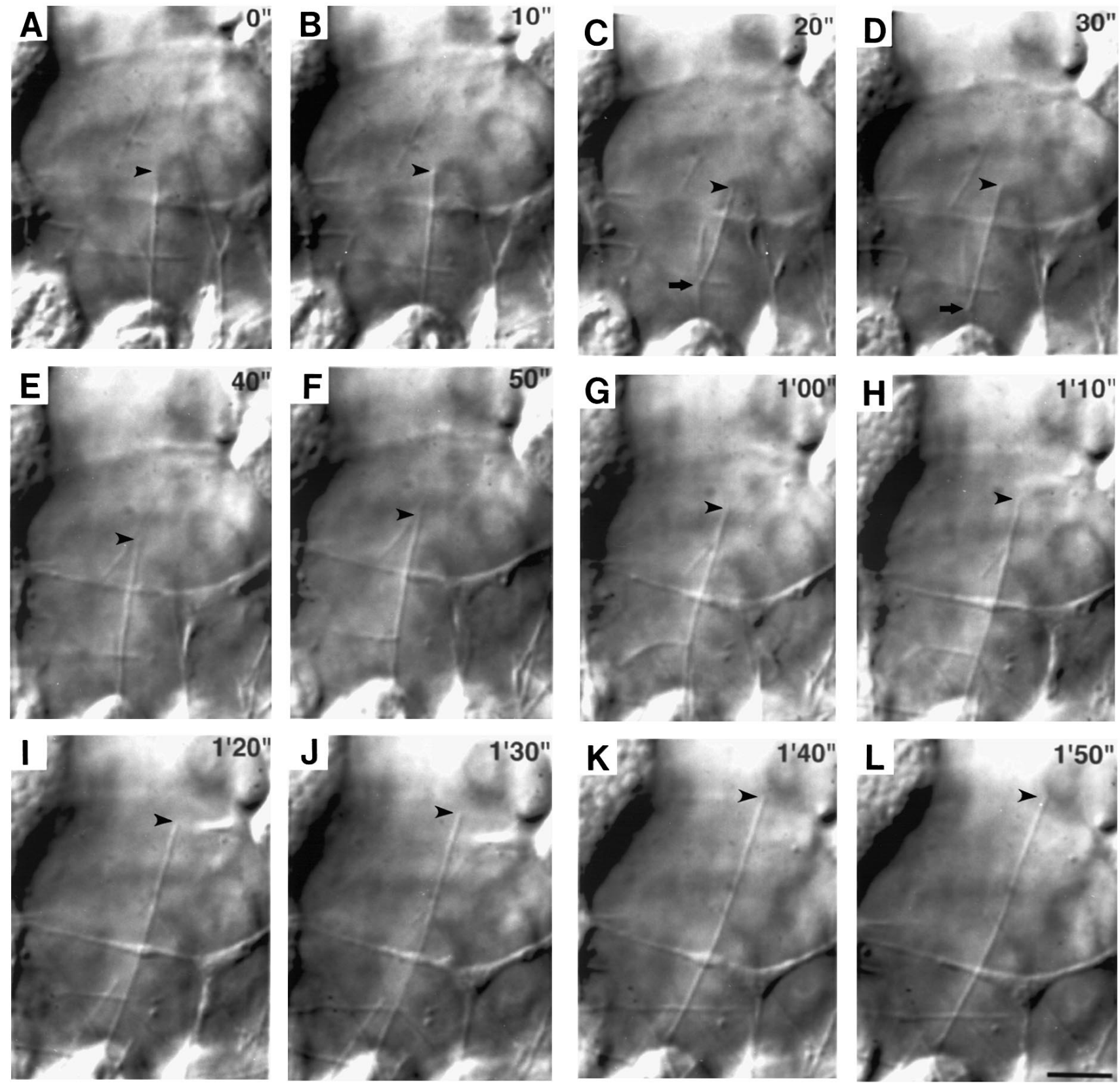

Fig. 6. Time-lapse sequence demonstrating the extension of a thin filopodium from a primary mesenchyme cell (A-L). An average growth rate for thin filopodia is around $10 \mu \mathrm{m} /$ minute. Rapid growth of $25 \mu \mathrm{m}$ has been observed on occasion. Sometimes the growth occurs at a constant uninterrupted rate and at other times growth is punctuated by pauses in the growth. An arrowhead marks the tip of the progressing filopodium in this sequence. At 10 seconds, the filopodium stops growing and a kink in the filopodium appears (arrow in C and D). At about 40 seconds, the filopodium begins growing again and grows at about $10 \mu \mathrm{m} /$ minute for the remainder of the sequence. Time elapsed from the first image is shown in the upper righthand corner in minutes and seconds. Scale bar, $5 \mu \mathrm{m}$.

To test if the nickel effect on filopodia was secondary to alterations of the ectoderm, PMCs were transferred from either control or from nickel-treated embryos into nickel or control ectodermal hulls. Nickel-treated PMCs extended thin filopodia of normal length in control ectoderm $(11.4 \pm 3 \mu \mathrm{m} n=11)$, a number that agreed well with the filopodia extended from normal filopodia in the ring. Untreated PMCs extended longer filopodia in nickel-treated ectoderm $(24.7 \pm 7 \mu \mathrm{m} n=14)$ and this number is conservative because many of the longer filopodia bent out of the plane of focus (Fig. 10). Thus positional cues in the environment play a significant role in filopodial behavior.

\section{The extracellular matrix is not essential for filopodial extension}

In the above experiments, filopodial length was influenced by the ectoderm and/or its underlying basal lamina. That influence could have been promoted by an altered extracellular matrix or it could have been due to other influences, including signals 

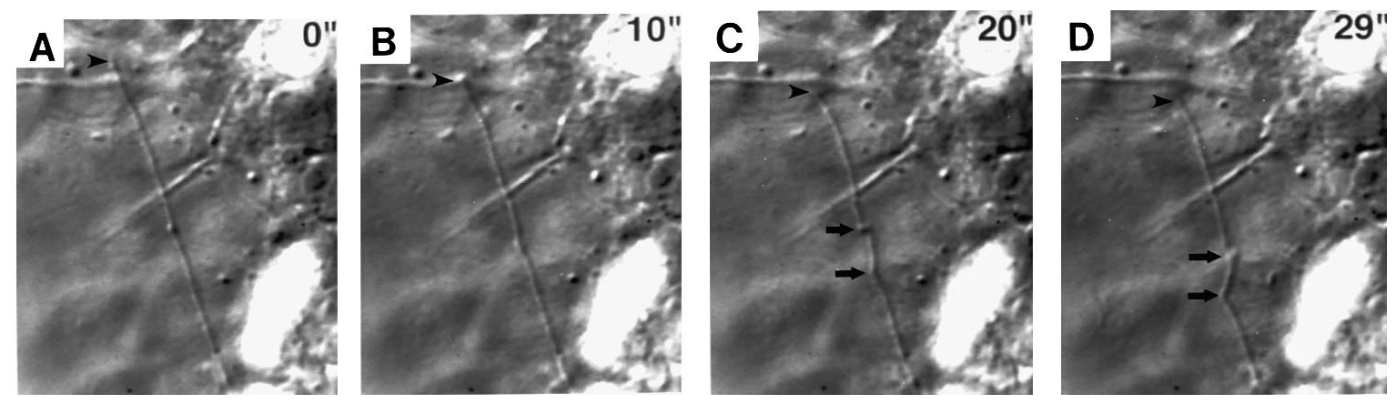

Fig. 7. Time-lapse sequence showing the retraction of a thin filopodium (A-L). An arrowhead marks the tip of the retracting filopodium. Just preceding and during retraction the filopodium is often observed to kink (arrows in $\mathrm{C}$ and D). The lifetime of a thin filopodium varies between 2 and about 30 minutes. Often a thin filopodium will be partially retracted and then will begin a new phase of extension. The filopodium depicted in this video sequence is retracted at about $11 \mu \mathrm{m} /$ minute which is close to the average rate of retraction. Time elapsed from the first image is shown in the upper righthand corner in minutes and seconds. Scale bar, $5 \mu \mathrm{m}$.
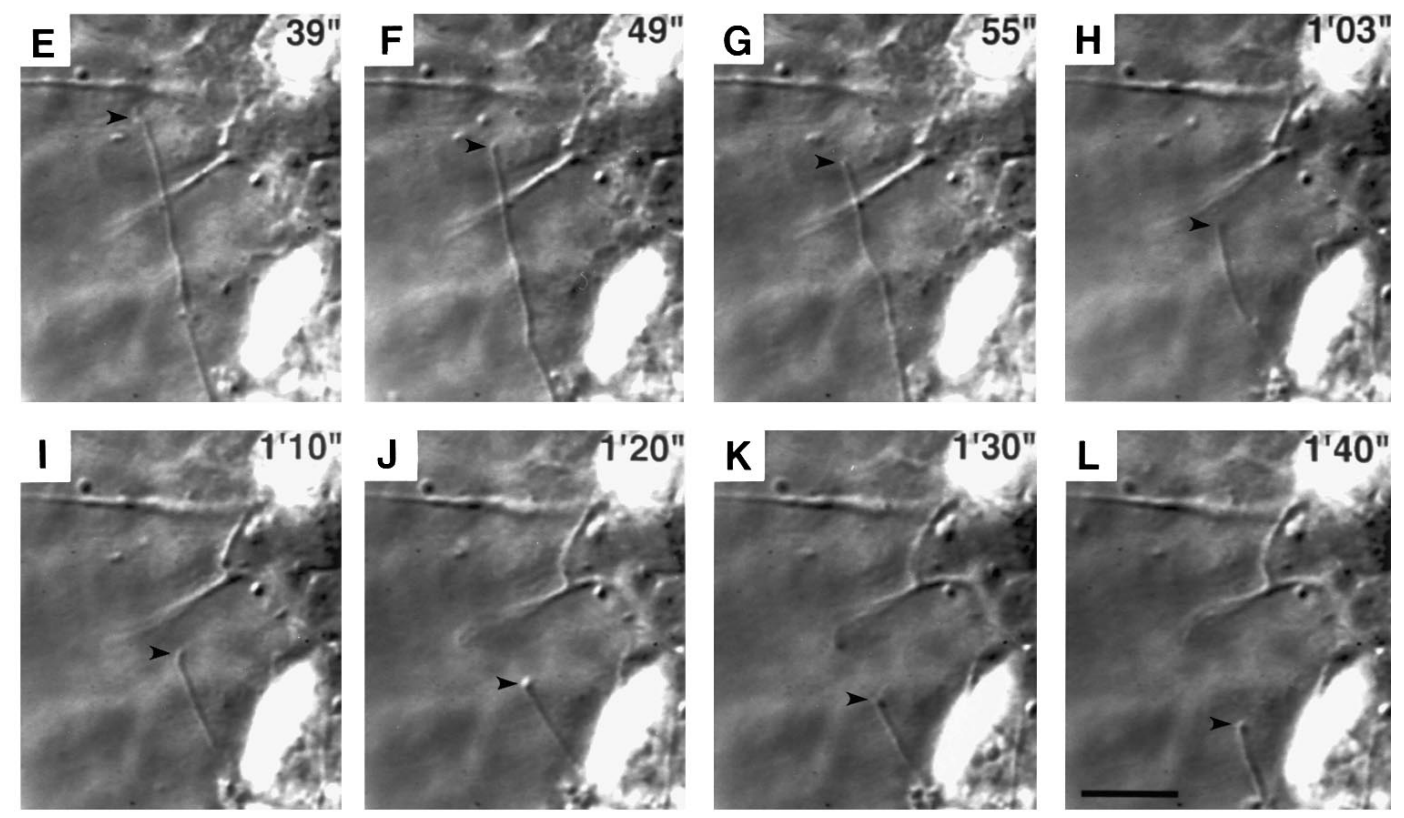

passing from the ectoderm to the PMCs via direct cell contact. To begin to distinguish between these possibilities, we perturbed the embryo by incubation in $\beta$-amino-proprio-nitrile ( $\beta$-APN), a lathrytic agent that prevents collagen crosslinking and severely impairs the deposition of the basal lamina (Wessel and McClay, 1987). Treatment of sea urchin embryos with $\beta$-APN prevents PMC migration; the treated embryos reach the mesenchyme blastula stage and PMCs remain quiescent at the vegetal pole. If the $\beta$-APN is washed out, even after one or two days, the embryos resume normal development. If left in $\beta$-APN for longer periods of time, the PMCs form highly abnormal stunted skeletons. We looked at thin filopodia in embryos treated with $\beta$-APN to determine if an intact basal laminar substratum was necessary for their extension. In treated embryos, even though the cells were not migrating, the PMCs extended thin filopodia.
Thus, even in the absence of cell motility and in the absence of an appropriate extracellular matrix, the filopodia are able to extend. This lends further support to the notion that thin filopodia are not tightly coupled with locomotion and it suggests that extracellular matrix is not the primary inducer of filopodial extension. While filopodia were present under continuous treatment, the longer thin filopodia, characteristic of the longitudinal cell chain, did not appear until the embryos were washed out of $\beta$-APN and had resumed normal development, including production of normal spicules.

\section{DISCUSSION}

Thin filopodia extended by PMCs, SMCs and ectodermal cells

Table 1. Comparison of filopod length in normal and $\mathrm{NiCl}_{2}$-treated embryos

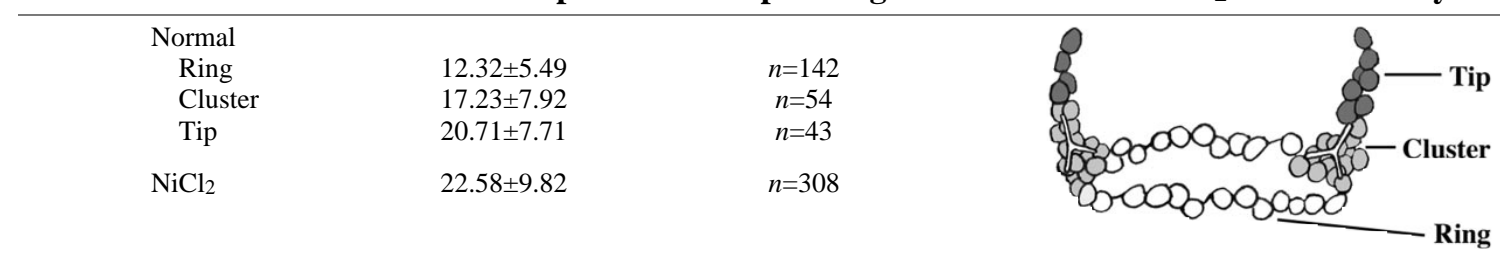

Individual filopods were measured from video sequences in which the entire length of the filopod was in the plane of focus. The diagram on the right illustrates the distribution of primary mesenchyme cells at the late gastrula stage and the regions used to determine if positional differences in filopod length exist in normal embryos. Values are given as average length \pm standard deviation. Differences in filopodial length are significant at the following levels: Ring versus Cluster $t$ $<0.0005$; Ring versus Tip $t<0.0005$; Cluster versus Tip $t<0.025$; Ring versus $\mathrm{NiCl}_{2} t<0.0005$; Cluster versus $\mathrm{NiCl}_{2} t<0.0005$; Tip versus NiCl $2 t<0.10$. 


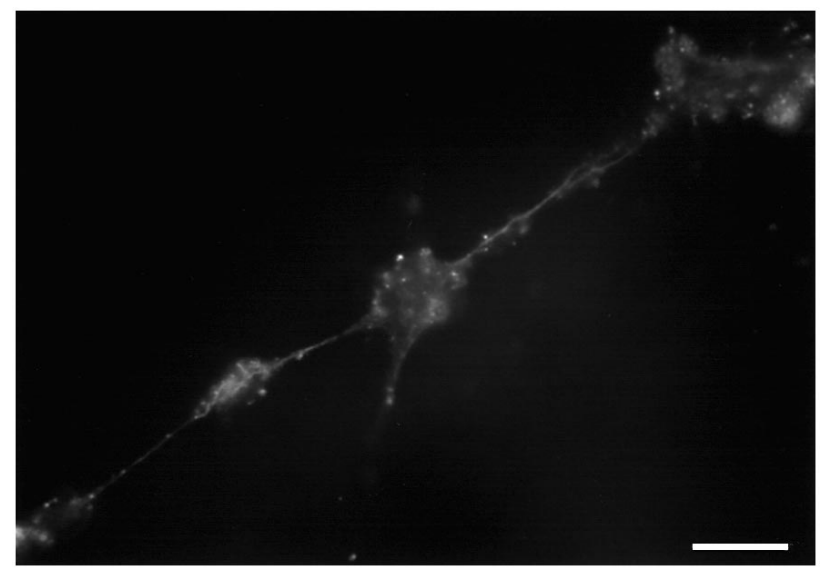

Fig. 8. Rhodamine-phalloidin staining of primary mesenchyme cells grown in culture demonstrates the presence of actin in thin filopodia. In culture, PMCs extend thin filopodia of the same dimension as those in vivo and each thin filopodium can be seen with a brightly stained bundle of actin filaments. Scale bar, $10 \mu \mathrm{m}$.

during gastrulation are highly dynamic with rapid growth and retraction rates. They grow at rates of up to $25 \mu \mathrm{m} /$ minute and thin filopodia extended by PMCs can grow to more than $80 \mu \mathrm{m}$ in length from cell bodies that are only about $8 \mu \mathrm{m}$ in diameter. Given the average extension length of the filopodia and the average size of the embryonic cells, we estimate that an individual PMC cell would have the opportunity to contact about 50 ectodermal cells without moving its cell body. At the tip of the longitudinal chain where the filopodia are even longer, the number of ectodermal cells within reach is even larger.

The thin filopodia produced by all of the different cells in the gastrulating sea urchin embryo have some significant similarities but some notable differences. The thin filopodia extended by PMCs and ectodermal cells are the most simple, varying little in diameter along their entire length. Occasionally, there is a bulge in a PMC filopodium that moves toward the cell body, and at times the tip of a PMC filopodium flattens onto a substratum. SMC thin filopodia are the same diameter as those from the PMCs but display more complex behavior; rather than remaining relatively uniform, these thin filopodia often thicken to become lamellipodia or thick filopodia. In contrast, the better characterized thick filopodia/lamellipodia that are extended by SMCs are significantly larger (1-3 $\mu \mathrm{m}$ in diameter) and taper from their base to their distal ends. These differences in thin filopodia are not due merely to changes in the extracellular environment, as neighboring PMCs and SMCs continue to display the differences, even when they are in direct contact. It is still not known if these differences in the dynamics of the cellular appendages produced by these two cells types reflect a difference in their functionality.

The structure of the thin filopodia pose some significant challenges in understanding both their function and assembly. The behavior and distribution of the filopodia suggest that they play a sensory role, thus requiring a means to convey information back from the filopodial tip to the cell body. Given that the filopodia are very thin $(200-400 \mathrm{~nm})$ and reach lengths far longer than $10 \mu \mathrm{m}$, diffusion would likely be too slow. An active process, perhaps an electrical event or a second messenger cascade, might provide the needed speed and gain. Alternatively, the retrograde transport, evidenced by the bulges that sometimes move from filopodial tip to cell body, may provide an active means to bring material and information about contacts at the tip. Transport is also an issue for the filopodia. Ultrastructurally, the thin filopodia appear to contain a sparse number of microtubules and an extensive network of actin filaments (Gibbins et al., 1969; Tilney and Gibbins, 1969; Karp and Solursh, 1985). The staining of sea urchin filopodia with rhodamine-phalloidin performed here provides further support for the notion that these structures are rich in F-actin. This requires that sufficient G-actin be transported along a 200 $\mathrm{nm}$ filament to the tip of the growing filopodium for assembly there, rapidly enough to support growth rates of up to 25 $\mu \mathrm{m} /$ minute. How G-actin concentrations are able to be maintained at a high enough level at the tip of the growing thin filopodium is not known but the mechanism must include an efficient transport of subunits.

A survey of other systems reveals that filopodial extensions, similar in dimension to those studied here, might be fairly
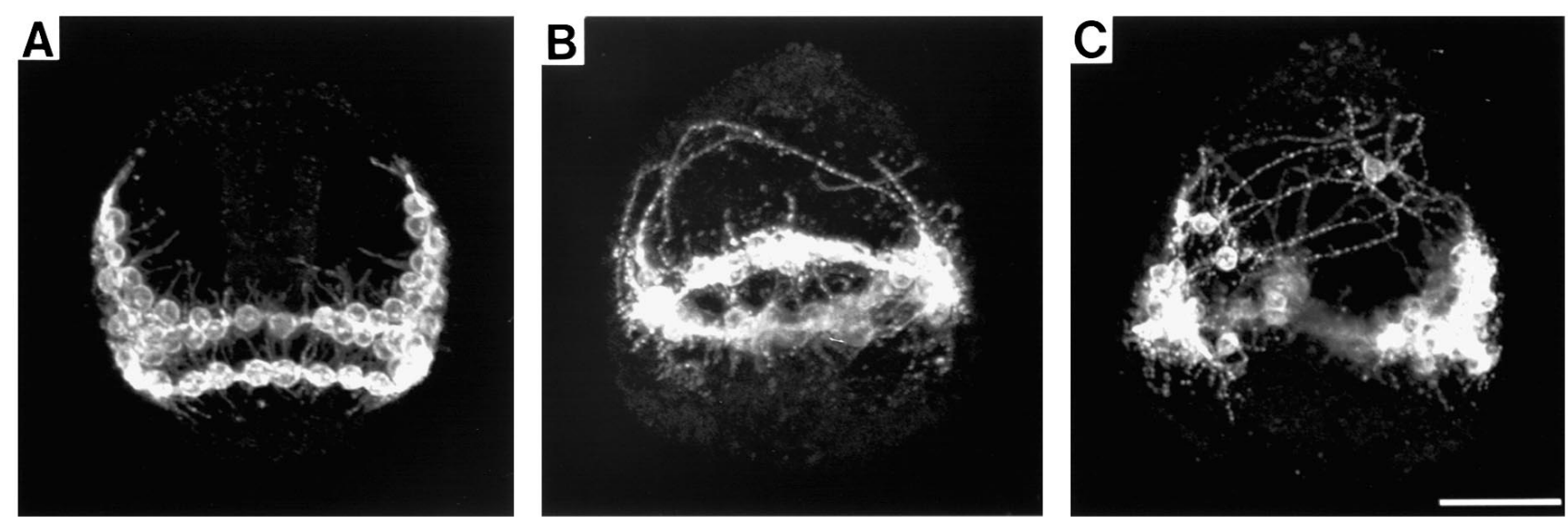

Fig. 9. Confocal images of PMCs stained with a monoclonal antibody specific for the cell surface of PMCs shows differences in filopodial extension between normal and $\mathrm{NiCl}_{2}$-treated embryos. Control embryos show an abundance of thin filopodia extending from the ring of PMCs (A). After treatment with $\mathrm{NiCl}_{2}$, which ventralizes the ectoderm, the thin filopodia are much longer (B,C) than in control embryos. Filopodia in $\mathrm{NiCl}_{2}$-treated embryos can reach lengths greater than $100 \mu \mathrm{m}$ and are often seen wrapping around the wall of the blastocoel. The antigen tends to aggregate during fixation of the thin filopodia to give them a slightly beaded appearance. Scale bar, $50 \mu \mathrm{m}$. 
common in a number of organisms. The strongest parallel might be drawn with the thin filopodia extended from neuronal growth cones. The diameters of growth cone filopodia in both vertebrates and invertebrates are similar to that reported here
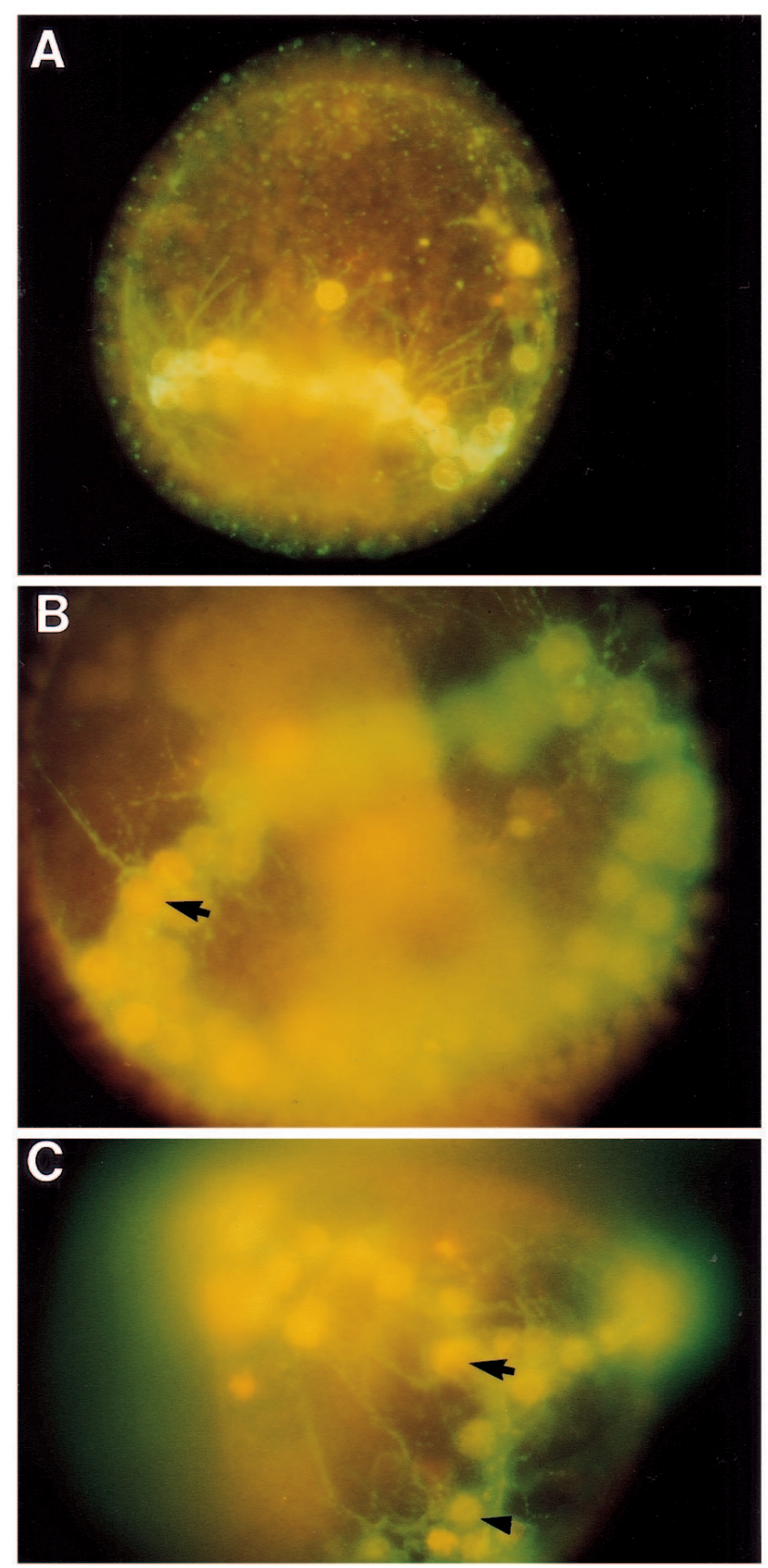

Fig. 10. Growth of thin filopodia is altered in recombinant embryos in which the ectoderm had been treated with $\mathrm{NiCl}_{2}$. In A-C, control PMCs (arrows in B and C) transferred to nickel-treated ectoderm hulls are shown to extend longer filopodia than they normally would have extended if left in their control environment. Each of the three embryos also is shown to have PMCs that are not red (arrowhead in C), indicating that they have PMCs converted from the SMC lineage, a phenomenon that allows for a quantitative restoration of the normal number of PMCs in an embryo (Ettensohn and McClay, 1988). In the reciprocal transfer, nickel-treated PMCs extended filopodia of normal length inside control ectodermal hulls.
(Taghert et al., 1982; Marsh and Letourneau, 1984). The extension and retraction rates of growth cone filopodia (Sheetz et al., 1990; Myers and Bastiani, 1993) also compare favorably. The rearward movement of membrane occurs in both, and the thin filopodia extending from growth cones do not appear to be necessary for movement of the growth cone (Marsh and Letourneau, 1984; Oakley and Tosney, 1993). Instead, a variety of evidence suggests that the growth cone thin filopodia act as chemosensors that influence indirectly the direction of growth cone migration (Taghert et al., 1982; Caudy and Bentley, 1986; Lemmon et al., 1992; Davenport et al., 1993; Oakley and Tosney, 1993). In fact, they have been described as 'long distance sensors of environmental cues that guide axons by influencing growth cone motility' (Oakley and Tosney, 1993). Similar to the arguments made here, indirect evidence suggests that the growth cone filopodia function correlates with cell interactions or cell signaling phenomena. For example, drug-treated growth cones, without filopodia, can advance but make dramatically larger numbers of errors. Thus, although there is no direct experimental proof in any system that thin filopodia play a purely sensory role, data from diverse systems are consistent with that model.

Several lines of indirect evidence suggest that sea urchin thin filopodia are involved in signaling between cells during gastrulation. First, neither the timing nor the localization of filopodial extension correlates with cell locomotion. The few filopodia that are present when PMCs move are not extended in any pattern that would suggest mechanical involvement in the locomotion. Second, there is circumstantial evidence showing that thin filopodia are present at locations and at times when cell-cell signaling is known to occur. They are present and highly enriched during the time when our previous experiments have shown PMCs interact with the ectoderm to obtain dorsal-ventral and animal-vegetal patterning information (Hardin et al., 1992; McClay et al., 1992; Armstrong et al., 1993; Armstrong and McClay, 1994). Furthermore, they are present between PMCs and SMCs at the time and in the locations where experimental evidence shows cell fate signaling to occur between these two cell types (cf. Ettensohn and McClay, 1988). Third, we present evidence that filopodial behavior is modified by changes in positional information provided by the ectoderm. Differences in filopodial extension were found to correlate with the position of PMCs in normal embryos and with the ventralization of ectoderm in $\mathrm{NiCl}_{2}-$ treated embryos. Reciprocal transplants of PMCs between normal and $\mathrm{NiCl}_{2}$-treated embryos confirmed that the observed differences in filopodial behavior are not cell autonomous but instead are substratum induced. None of these results alone can prove that the thin filopodia are involved in cell-signaling events; however, taken together, the findings demonstrate that they are responsive to positional information and that they could provide a direct substratum for cell interaction over some distance during gastrulation.

If it is correct that the filopodia are involved in obtaining positional information needed for pattern formation, then several aspects of the positional models will have to be rethought. Many cell-cell signals that have been characterized to date in the sea urchin occur over some distance between cells that are not near neighbors. One popular interpretation of these observations, originally proposed by Runnstrom (1929), is that the assignment of positions occurs by diffusion of signals to 
create gradients. However, given the reach of thin filopodia and the size of the embryo, the potential sampling range for each cell can be over a distance that may encompass more than 50 cells. Thus, the long range of the filopodia may offer a means for short-range ligand-receptor systems to act at a distance. The extended range of sampling and the increased reliability that results may call for a reappraisal of some of the current models of pattern formation.

The thin filopodia in the sea urchin embryo should provide a fruitful setting for studies of the form and function of filopodia. Studies of filopodial mechanics are made possible by the ability to observe the thin filopodia in the intact embryo and to predict their presence at defined locations in the embryo. Furthermore, the rich experimental literature on the sea urchin embryo provides a significant arsenal of tools for testing mechanistic hypotheses about their role in development. The combination of predictable cell behaviors that can be observed readily, with well-established tools for manipulating embryonic pattern, will permit detailed analyses of the dynamics of the filopodial structures, their mechanism for signal transduction and their role in embryogenesis.

This collaboration grew from observations that were made during the Embryology Course at the Marine Biological Laboratory, Woods Hole. The authors appreciate the input from the students and faculty of that course. This work was supported by NIH grant HD14483 to DRM and by NIMH Silvio Conti Center grant MH49176 to SF.

\section{REFERENCES}

Armstrong, N., Hardin, J. and McClay, D. R. (1993). Cell-cell interactions regulate skeleton formation in the sea urchin embryo. Development 119, 833840 .

Armstrong, N. and McClay, D. R. (1994). Skeletal pattern is specified autonomously by the primary mesenchyme cells in sea urchin embryos. Dev. Biol. 162, 329-338.

Caudy, M. and Bentley, D. (1986). Pioneer growth cone steering along a series of neuronal and non-neuronal cues of different affinities. J. Neurosci. 6, 1781-1795.

Davenport, R. W., Dou, P., Rehder, V. and Kater, S. B. (1993). A sensory role for neuronal growth cone filopodia. Nature 361, 721-724.

Ettensohn, C. A. and McClay, D. R. (1986). The regulation of primary mesenchyme cell migration in the sea urchin embryo: transplantations of cells and latex beads. Dev. Biol. 117, 380-391.

Ettensohn, C. A. and McClay, D. R. (1988). Cell lineage conversion in the sea urchin embryo. Dev. Biol. 125, 396-409.

Ettensohn, C. A. (1990a). Cell interactions in the sea urchin embryo studied by fluorescence photoablation. Science 248, 1115-1118.

Ettensohn, C. A. (1990b). The regulation of primary mesenchyme cell patterning. Dev. Biol. 140, 261-271.

Gibbins, J. R., Tilney, L. G. and Porter, K. R. (1969). Microtubules in the formation and development of the primary mesenchyme in Arbacia punctulata. I. The distribution of microtubules. J. Cell Biol. 41, 201-226.

Gustafson, T. and Wolpert, L. (1961). Studies on the cellular basis of morphogenesis in the sea urchin embryo; directed movements of primary mesenchyme cells in normal and vegetalized larvae. Exp. Cell Res. 24, 64-79.

Gustafson, T. (1963). Cellular mechanisms in the morphogenesis of the sea urchin embryo. Cell contacts within the ectoderm and between mesenchyme and ectoderm cells. Exp. Cell Res. 32, 570-589.

Gustafson, T. (1964). The role and activities of pseudopodia during morphogenesis of the sea urchin larva. In Primitive Motile Systems, (eds. R. D. Allen and N. Kamiya), pp. 333-349. Orlando: Academic Press.

Gustafson, T. and Wolpert, L. (1967). Cellular movement and contact in sea urchin morphogenesis. Biol. Rev. 42, 442-498.

Hardin, J. D. and Cheng, L. Y. (1986). The mechanisms and mechanics of archenteron elongation during sea urchin gastrulation. Dev. Biol. 115, 490501.

Hardin, J. D. (1987). Archenteron elongation in the sea urchin embryo is a microtubule-independent process. Dev. Biol. 121, 253-262.

Hardin, J. (1988). The role of secondary mesenchyme cells during sea urchin gastrulation studied by laser ablation. Development 103, 317-324.

Hardin, J., Coffman, J. A., Black, S. D. and McClay, D. R. (1992). Commitment along the dorsoventral axis of the sea urchin embryo is altered in response to $\mathrm{NiCl} 2$. Development 116, 671-685.

Hardin, J. and McClay, D. R. (1990). Target recognition by the archenteron during sea urchin gastrulation. Dev. Biol. 142, 86-102.

Karp, G. C. and Solursh, M. (1985). Dynamic activity of the filopodia of sea urchin embryonic cells and their role in directed migration of the primary mesenchyme in vitro. Dev. Biol. 112, 276-283.

Leaf, D. S., Anstrom, J. A., Chin, J. E., Harkey, M. A. and Raff, R. A. (1987). Antibodies to a fusion protein identify a cDNA clone encoding msp130, a primary mesenchyme-specific cell surface protein of the sea urchin embryo. Dev. Biol. 121, 29-40.

Lemmon, V., Burden, S. M., Payne, H. R., Elmslie, G. W. and Hlavin, S. (1992). Neurite growth on different substrates: permissive versus instructive influences and the role of adhesive strength. J. Neurosci 12, 818-826.

Marsh, L. and Letourneau, P. C. (1984). Growth of neurites without filopodial or lamellapodial activity in the presence of cytochalasin B. J. Cell Biol. 99, 2041-2047.

McClay, D. R., Cannon, G. W., Wessel, G. M., Fink, R. D. and Marchase, R. B. (1983). Patterns of antigenic expression in early sea urchin development. In Time, Space, and Pattern in Embryonic Development (eds. W. R. Jeffrey and R. A. Raff), pp. 157-169. New York: Alan R. Liss.

McClay, D. R., Armstrong, N. A. and Hardin, J. (1992). Pattern formation during gastrulation in the sea urchin embryo. Development 1992 Supplement, 33-41.

Myers, P. Z. and Bastiani, M. J. (1993). Growth cone dynamics during the migration of an identified commisural growth cone. J. Neurosci. 13, 127-143.

Oakley, R. A. and Tosney, K. W. (1993). Contact-mediated mechanisms of motor axon segmentation. J. Neurosci 13, 3773-3792.

Runnstrom, J. (1929). Uber selbstifferenzierung und induktion bei dem seeigelkeim. Wilhelm Roux Arch. EntwMech. Org. 117, 123-145.

Sheetz, M. P., Baumrind, N. L., Wayne, D. B. and Pearlman, A. L. (1990). Concentrations of membrane antigens by forward transport and trapping in neuronal growth cones. Cell 61, 231-241.

Sheetz, M. P., Wayne, D. B. and Pearlman, A. L. (1992). Extension of filopodia by motor-dependent actin assembly. Cell Motil.Cytoskeleton 22, 160-169.

Taghert, P. H., Bastiani, M. J., Ho, R. K. and Goodman, C. S. (1982). Guidance of pioneer growth cones: filopodial contacts and coupling revealed with an antibody to lucifer yellow. Dev. Biol. 94, 391-399.

Tilney, L. G. and Gibbins, J. R. (1969). Microtubules and filaments in the filopodia of the secondary mesenchyme cells of Arbacia punctulata and Echinarachnius parma. J. Cell Sci. 5, 195-210.

Wessel, G. M. and McClay, D. R. (1987). Gastrulation in the sea urchin embryo requires the deposition of crosslinked collagen within the extracellular matrix. Dev. Biol. 121, 149-165. 